\title{
A novel measuring system for the metrological characterization of piezoresistive films at high temperature
}

\author{
Damiano Crescini, and Marco Romani \\ Dipartimento di Elettronica per l'Automazione \\ Facoltà di Ingegneria - Università di Brescia - Via Branze 38 - 25123 Brescia - Italy \\ Tel. +39030 3715547 - Fax +39030 380014 \\ EMAIL: damiano.crescini@ing.unibs.it
}

\begin{abstract}
In different industrial applications, such as injection molding and/or hot rolling, it is necessary to measure pressure in critical environmental conditions at high temperature. A promising approach for high temperature applications is the use of a wide band gap semiconductor such as silicon carbide (SiC). The excellent electrical and mechanical properties as temperature far beyond $350^{\circ} \mathrm{C}$ make it a suitable material for electromechanical sensors in high temperature application. The purpose of this work was to investigate the possibility of developing a testing structure to evaluate the longitudinal and transverse guage factors $\left(\mathrm{GF}_{\mathrm{L}}\right.$ and $\left.\mathrm{GF}_{\mathrm{T}}\right)$ in piezoresistive films in $\mathrm{SiC}$, to evaluate the TCR and finally to define the temperature effect up to $400^{\circ} \mathrm{C}$. In the following paper a tailored test structure has been presented together with the preliminary results obtained from piezoeristors realized in $\mathrm{SiC}$ technology.

The solution proposed assures the most accurate measurement of the parameters of the film without any undesirable influence.
\end{abstract}

\section{Introduction}

In different industrial applications, such as injection molding and/or hot rolling, it is necessary to measure pressure in critical environmental conditions where the temperature can reach values in the range of 350$380^{\circ} \mathrm{C}$.

In these cases, a fluid with low thermal conductivity is now typically used in order to transfer the pressure to the sensitive element, while preserving it from overheats. A preferred solution would be that of obtaining new sensing elements with an intrinsic capability of operation over an extended temperature range.

Silicon piezoresistors are unsuitable for use in high-temperature applications due to the significant decrease of the piezoresistive effect in this material with rising temperature. On the other hand, piezoresistive films in SiC has been recently investigated as a material exhibiting a piezoresistive effect, which appears to be exploitable at high temperature [1-3].

The purpose of this work is, therefore, to investigate the possibility of developing a testing structure to evaluate the longitudinal and transverse guage factors $\left(G F_{L}\right.$ and $\left.G F_{T}\right)$ in piezoresistive films in $S i C$, to evaluate the TCR and finally to define the temperature effect up to $400^{\circ} \mathrm{C}$.

\section{The test structures}

The piezoresistive films in SiC deposited on silicon test structures were tested by measuring their resistance and gage factor at up to $400^{\circ} \mathrm{C}$ by means of a purposely-designed test apparatus.

For the geometry of the test structures, a two-point simply supported beam was chosen. This solution assures the most accurate measurement of the parameters of the film without any undesirable influence of the clamping constraints and of their changes due to temperature.

In figure 1 a sketch of the testing apparatus is presented where the following parts are reported:

- Triangulation laser displacement sensor for specimens deflection measurement

- Infrared sensor adopted to reveals surface temperature variations of the specimens under test

- Loading head constituted by a hardened steel knife (or ceramic)

a Microprobes to connect the external instrumentation with the chip under test 
W Wire bonding between the thick film conductor on ceramic and the chip pads

Fig. 2 shows a detail of the ceramic support adopted for the clamping condition and for the heating process. The support has been realized on alumina substrate and sensibilized by using the thick film technology (for the heater and for the output connectors).

A displacement actuator is used to deflect the beam and induce a known amount of strain on the film. To this purpose, a piezoelectric stack was chosen with an internal position feedback provided by a built-in estensimetric sensor. The actuator plunger terminates with a ceramic knife-edge that generates a stress on the beam supporting the piezoresistive film. The stress causes a strain on the film and, in turn, a variation of its resistance, which is measured.

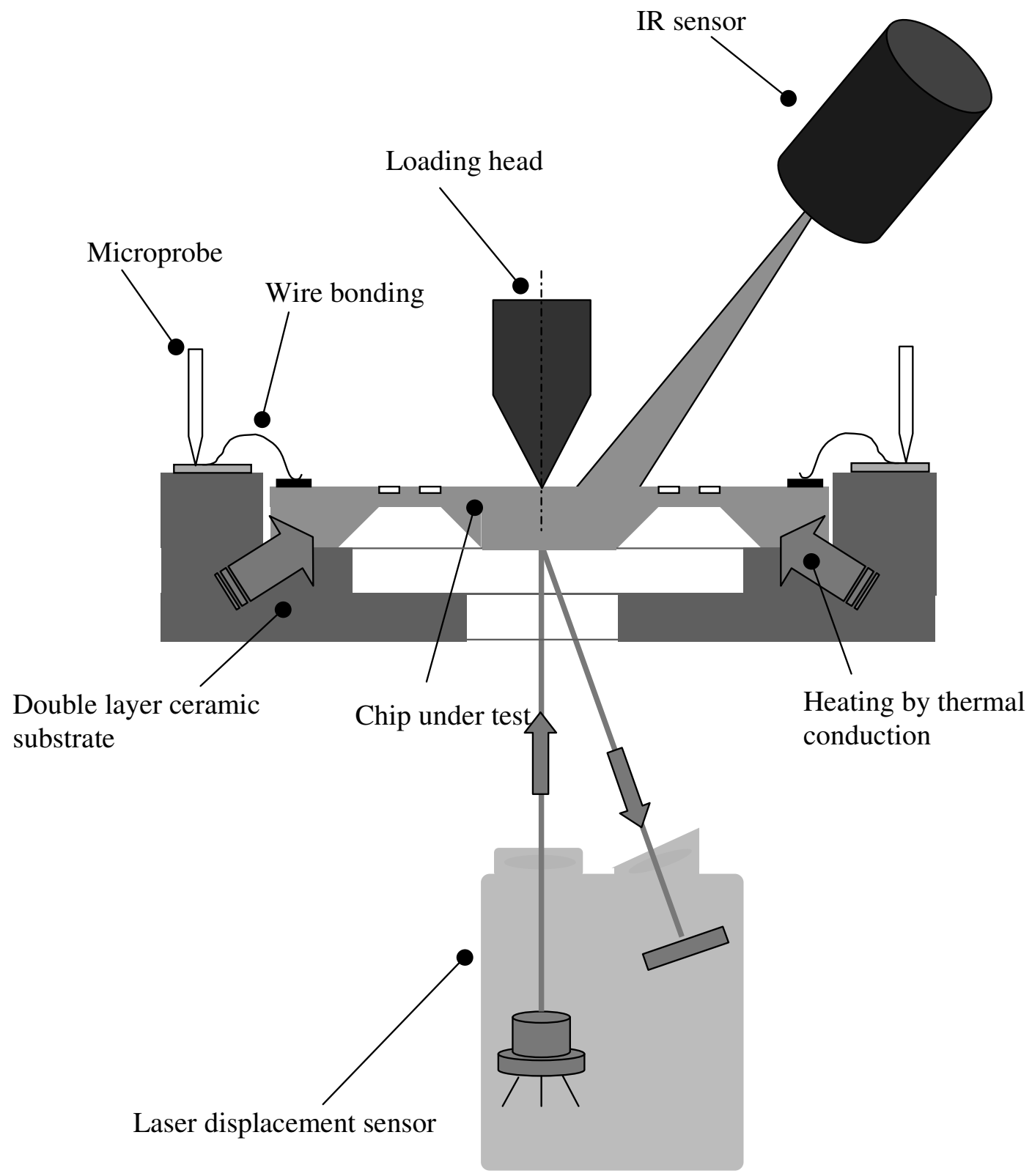

Figure 1 - Sketch of the testing apparatus 
The heating of the sample takes place by means of heaters realized in thick-film technology. In order to determine accurately the temperature of the substrate under test it is necessary to avoid of directly contacting it, otherwise the measurement could be affected by thermal loading. Therefore, a pyrometer sensor has been adopted. A position sensor to measure the beam deflection has been inserted in the supporting base. The sensor is a laser triangulation. The setting of all the sensors and the sample loading condition is obtained by means of a power amplifier, which communicates with a PC-based data acquisition system. The acquisition system enables to perform automated test cycles and collect data for up to 48 hours. Concurrently with the experimental characterization of the test structures, the design of the sensor diaphragm is also being optimized with the help of FEM simulations.
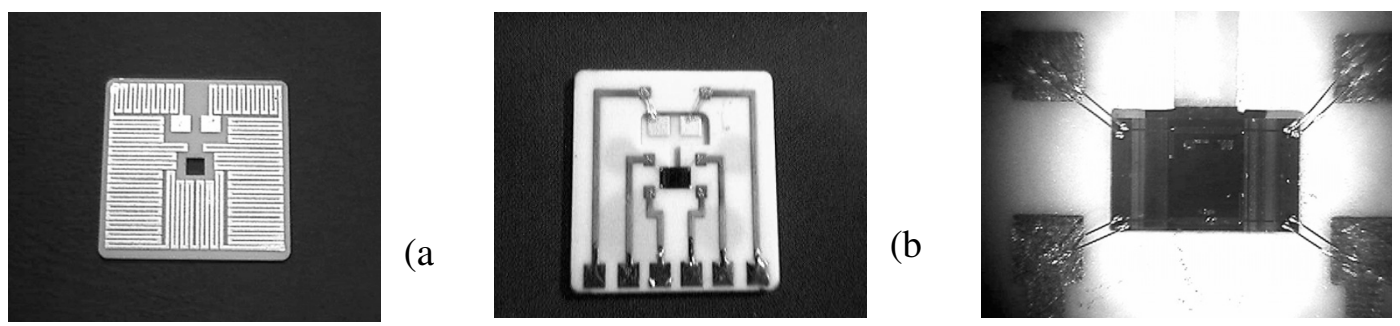

(c

Figure 2 - (a) Photograph of the heating element in thick-film technology on ceramic (b) the full older with the terminations (c) an enlargement of the chip in $3 \mathrm{C}-\mathrm{SiC}$ with bonding

Fig. 3 shows photograph of the testing structure. In figure 4 is reported an enlargement of the previous photograph with the aim to show: the microprobes for the external connection, the loading head and the chip under test.

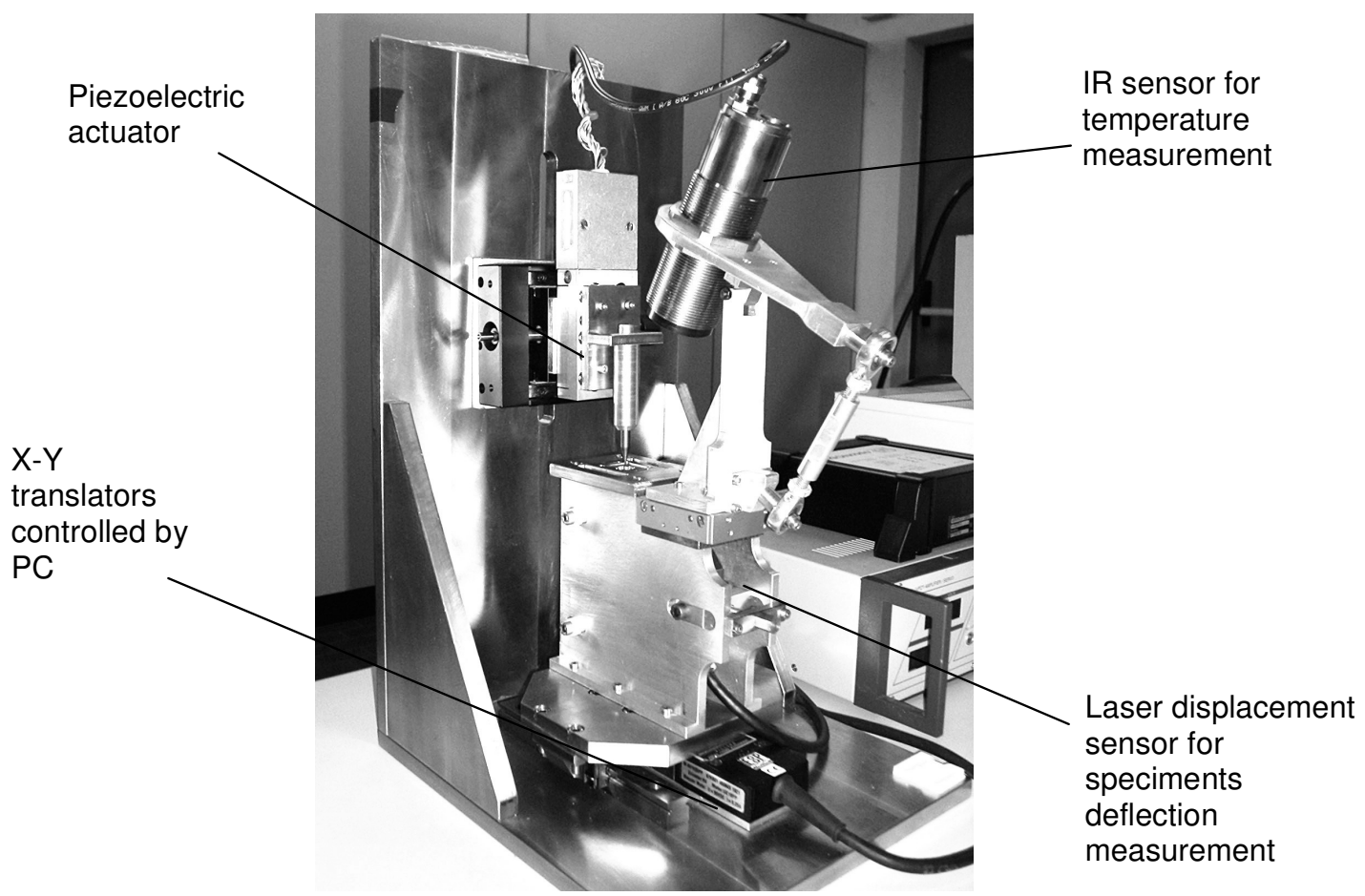

Figure 3 - Photograph of the testing structure 


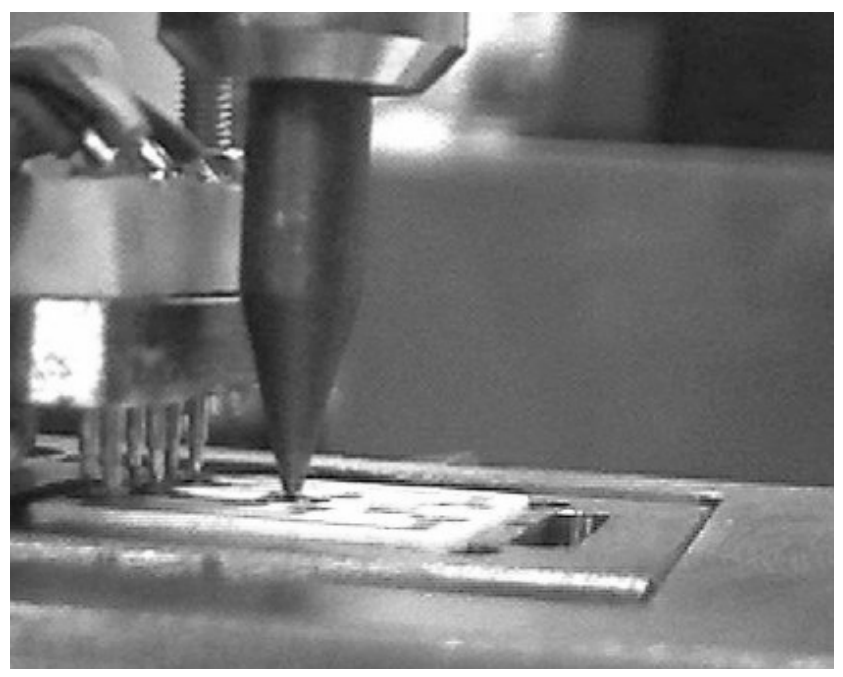

Figure 4 - Photograph of the loading head, microprobes and chip under test

\section{Preliminary experimental results}

The slope of the Vout from the chip (Wheatstone bridge output) vs. diaphragm deflection is the sensitivity. A typical value of about $0.35 \mathrm{mV} /$ microns has been found. Fig 5 shows the bridge input resistance change with temperatures up to $350^{\circ} \mathrm{C}$. The gauge factor of the piezoresistors changes with elevating temperature. A typical result is reported in Fig. 6. The gauge factor decreases with increasing the temperature up to $350^{\circ} \mathrm{C}$.

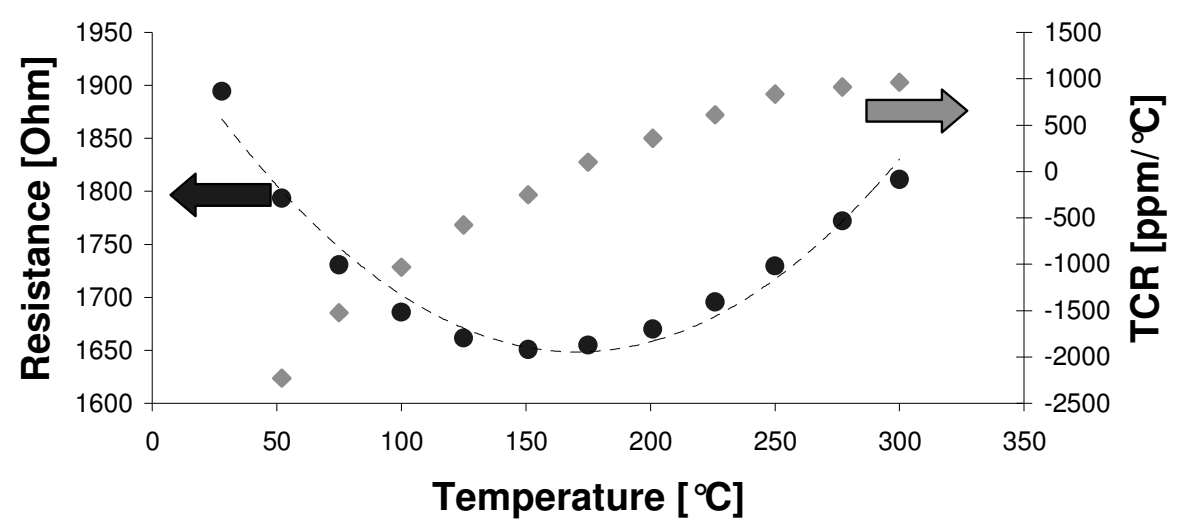

Figure 5 - Bridge Resistance Change With Temperature 


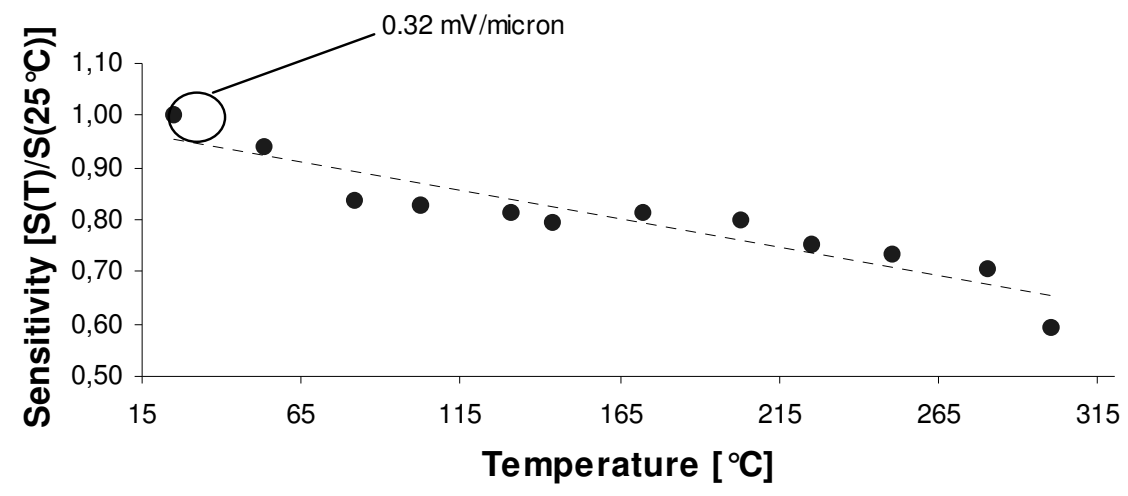

Figure 6 - Sensitivity behaviour of the piezoresistive elements connected in a Wheatstone bridge vs. temperature obtained with the testing structure. The deflection of the diaphragm is 10 microns

\section{Conclusions}

The purpose of this work was to investigate the possibility of developing a testing structure to measure the longitudinal and transverse guage factors $\left(\mathrm{GF}_{\mathrm{L}}\right.$ and $\left.\mathrm{GF}_{\mathrm{T}}\right)$ in piezoresistive films in $\mathrm{SiC}$, to evaluate the TCR and finally to define the temperature effect up to $400^{\circ} \mathrm{C}$.

In the following paper a dedicated test structure has been presented together with the preliminary results obtained from piezoresistors realized in $\mathrm{SiC}$ technology.

The solution proposed assures the most accurate measurement of the parameters of the film without any undesirable influence. Future improvements will concentrate on the heating process with the aim to test chip up to $500{ }^{\circ} \mathrm{C}$.

\section{Acknowledgements}

The authors acknowledge the contributions of the research partners in the project: MIUR (the Italian Ministry of Education, University and Research) and GEFRAN SENSORI Spa.

\section{References}

[1] T. G. Brown, B. Davis, D. Hepner, J. Faust, C. Myers,C. Muller, T. Harkins, M. Holis, and B. Placzankis, "Strap-Down Microelectromechanical (MEMS) Sensors for High-G Munition Applications,"IEEE Trans. On Magnetics, vol: 37 No. 1, pp. 336-342, Jan. 2001.

[2] G. L. Katulka, D. J. Hepner, B. Davis, E. Irwin, M.Ridgley, and K. Kornegay, "Characterization of Silicon Carbide and Commercial-Off-The-Shelf (COTS)Components for High-g Launch and EM Applications," IEEE Trans on Magnetics, vol: 37 No.1, pp. 248-251, Jan. 2001.

[3] J. C. Greenwood, "Silicon in Mechanical Sensors," J. Phys. E., Sci. Instrum., 21, pp. 1114-1128, 1988. 